\title{
BEM AND FEM ANALYSIS OF THE FLUID-STRUCTURE INTERACTION IN TANKS WITH BAFFLES
}

\author{
V. GNITKO, K. DEGTYARIOV, V. NAUMENKO \& E. STRELNIKOVA \\ A.N.Podgorny Institute for Mechanical Engineering Problems of the Ukrainian Academy of Sciences, Ukraine.
}

\begin{abstract}
In this paper we consider vibrations of the baffled elastic fuel tank partially filled with a liquid. The compound shell was a simplified model of a fuel tank. The shell is considered to be thin and the Kirchhoff-Love linear theory hypotheses are applied. The liquid is supposed to be an ideal and incompressible one and its flow introduced by the vibrations of a shell is irrotational. The problem of the fluid-structure interaction was solved using the reduced boundary and finite element methods. The tank structure was modeled by the FEM and the liquid sloshing in a fluid domain was described by using the multi-domain BEM. The rigid and elastic baffled tanks of different forms were considered. The dependencies of frequencies via the filling level were obtained numerically for vibrations of the fluid-filled tanks with and without baffles.

Keywords: baffles, fluid-structure interaction, free vibrations, liquid sloshing, multi-domain boundary element method, systems of singular integral equations.
\end{abstract}

\section{INTRODUCTION}

In fluid dynamics, the sloshing is defined as the motion of the free surface of a liquid in a partially filled tank or container. The computation of dynamic slosh forces arising due to the liquid motion within a partially filled tank is quite important in analyzing the directional behavior of tank trucks during various highway maneuvers. This problem is common in fuel tanks of automobiles, aircrafts, large ships and tankers.

When liquids slosh in closed containers, one can observe the multiple configurations (modes) in which the surface may evolve. Commonly, the different modes can be defined by their wave number $\alpha$ (number of waves in the circumferential direction) and by their mode number $n$.

Although baffles are commonly used as the effective means of suppressing the sloshing magnitudes, the only few studies have assessed the role of baffle design factors. The size and location effects of a baffle orifice on the sloshing has been reported in only two studies devoted rectangular Popov et al. [1] and generic Guorong and Rakheja [2] cross-section tanks. It should be noted that anti-slosh properties of baffle designs have been investigated through laboratory experiments by using small size tanks of different geometry Lloyd et al. [3].

The overview of the research on the topic [2-5] demonstrates that the dynamic response of liquid-containing structures can be significantly influenced by vibrations of their elastic walls in interaction with the sloshing liquid.

The most of researchers have described the fluid-structure interaction neglecting gravity or elasticity effects. The considerable results were obtained in [4, 5]. Bermudez et al. [4] studied in vibrations of the $2 \mathrm{D}$ elastic vessel partially filled with an incompressible fluid under the gravity force. Here the only 2D rectangular tank was under consideration. The research work of Gavrilyuk et al. [5] was devoted to the vibration analysis of baffled cylindrical shells, but both shells and baffles were rigid. In this work the authors developed the analytical method. So there are some limitations in these methods, and a further study is needed for each new form of tanks. 
With respect to the all numerical work, which has been done, it is fair to say that there is still no fully efficient numerical method to deal with the sloshing in elastic fluid-filled baffled tanks. The novelty of the proposed approach consists in possibility to study the influence of both rigid and elastic baffles in the fluid-filled tanks in the form of shells of revolution with an arbitrary meridian.

\section{PROBLEM STATEMENT}

Consider the problem of free harmonic vibrations of the fluid-filled elastic shell of revolution having an arbitrary meridian, with internal baffles installed to damp the liquid sloshing. The shell is of uniform thickness $h$, and height $L$, made of homogeneous, isotropic material with elasticity modulus $E$, Poisson's ratio $v$ and mass density $\rho_{s}$. The shell structure and its sketch are shown in Figure 1.

Denote the wetted part of the shell surface through $\sigma$ and the free surface of a liquid as $S_{0}$. The liquid volume was divided here into two domains. The shell surface $\sigma$ consists of four parts, $\sigma=S_{1} \cup S_{2} \cup S_{\text {bot }} \cup S_{\text {baf }}$. Here $S_{1}$ and $S_{2}$ are lateral surfaces of first and second fluid domains, respectively, $S_{\text {bot }}$ is the surface of the tank bottom and $S_{\text {baf }}$ is the baffle surface.

Let $\mathbf{U}=\left(U_{1}, U_{2}, U_{3}\right)$ denote the vector-function of shell displacements.

Consider at first stage the free vibrations of the shell without a liquid (the empty shell). Assume that the time dependent shell displacements are given by

$$
\mathrm{U}=\mathrm{u} \exp (i \Omega t) ; \mathrm{u}=\left(u_{1}, u_{2}, u_{3}\right) .
$$

Here $\Omega$ is the vibration frequency; the time factor $\exp (i \Omega)$ will be omitted further on. After the separation of the time factor, the vibrations of the shell without a liquid are described by the system of three partial differential equations

$$
\sum_{i=1}^{3} L_{i j} u_{i}=\Omega^{2} u_{j}, j=1,2,3,
$$

where $L_{i j}$ are linear differential operators of Kirchhoff-Love shell theory Levitin and Vassiliev [6].

The finite element method was applied by Ventsel et al. [7] to evaluate the natural frequencies $\Omega_{k}$ and modes $\mathbf{u}_{k}, k=\overline{1, N}$ of the shell of revolution without a liquid. After forming the
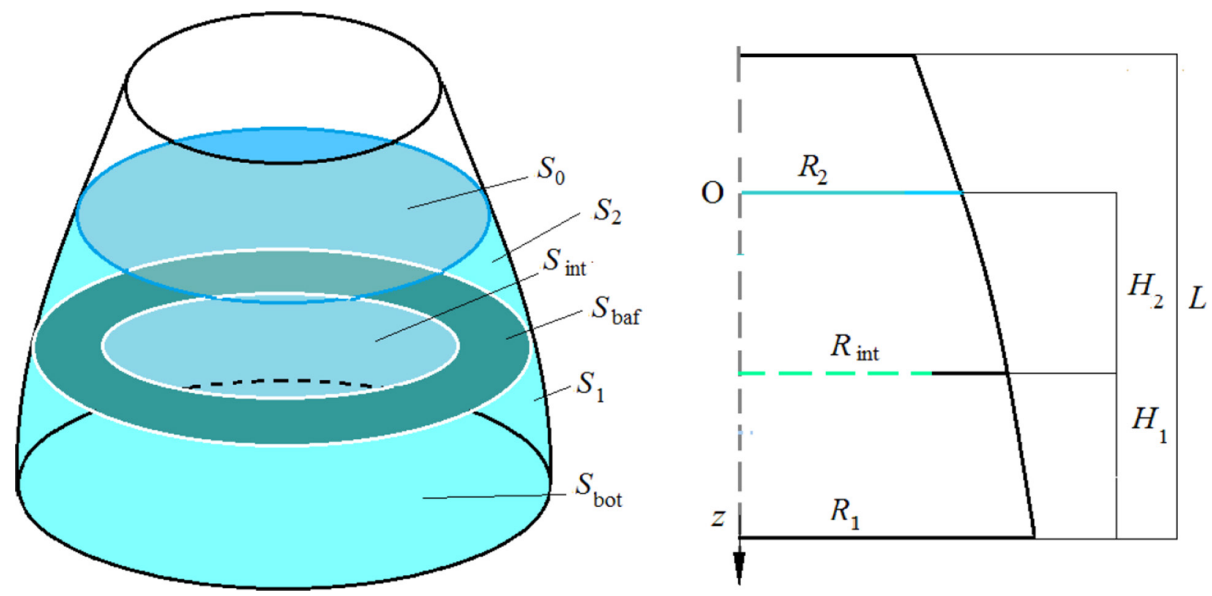

Figure 1: Shell structure with an internal baffle. 
global stiffness $\mathbf{L}$ and mass $\mathbf{M}$ matrices, the following equation of motion for the shell containing fluid was obtained in [7]:

$$
\mathrm{LU}+\mathrm{MU}=p_{d} \mathrm{n},
$$

where $\mathbf{n}$ is an external unit normal to the shell wetted surface, the term $p_{d} \mathbf{n}$ gives the fluid dynamical pressure upon the shell, normal to its surface. Note that the following equalities are valid for each eigenvalue and eigenmode

$$
\mathrm{Lu}_{k}=\Omega_{k}^{2} \mathrm{MU}_{k}, \quad\left(\mathrm{MU}_{k}, \mathrm{u}_{j}\right)=\delta_{k j} .
$$

To model the fluid domain, a mathematical model has been developed based on the following hypotheses: the fluid is incompressible, the motion of the fluid is irrotational and inviscid, only small vibrations (linear theory) need to be considered. So a scalar velocity potential $\Phi(x, y, z, t)$ whose gradient represents the fluid velocity can be introduced.

The fluid pressure $p=p(x, y, z, t)$ acting on the wetted shell surface is obtained from the linearized Bernoulli's equation for a potential flow Lamb [8]

$$
p=-\rho_{l}\left(\frac{\partial \Phi}{\partial t}+g z\right)+p_{0}, p_{s}=-\rho_{l} g z, p_{d}=-\rho_{l} \frac{\partial \Phi}{\partial t},
$$

where $g$ is the gravity acceleration, $z$ is the vertical coordinate of a point in the liquid, $\rho_{l}$ is the density of a liquid, $p_{s}$ and $p_{d}$ are static and dynamic components of the fluid pressure, $p_{0}$ is for atmospheric pressure.

At any instant the velocity potential $\Phi(x, y, z, t)$ could be determined from the following boundary value problem:

$$
\nabla^{2} \Phi=\frac{\partial^{2} \Phi}{\partial x^{2}}+\frac{\partial^{2} \Phi}{\partial y^{2}}+\frac{\partial^{2} \Phi}{\partial z^{2}}=0,\left.\frac{\partial \Phi}{\partial \mathrm{n}}\right|_{\sigma}=\frac{\partial w}{\partial t},\left.\frac{\partial \Phi}{\partial \mathrm{n}}\right|_{s_{0}}=\frac{\partial \zeta}{\partial t} ; \frac{\partial \Phi}{\partial t}+\left.g \zeta\right|_{s_{0}}=0
$$

where $w$ denotes the normal component of the displacement vector $\mathbf{U}$, namely, $w=(\mathrm{U}, \mathrm{n})$; the unknown function $\zeta=\zeta(x, y, t)$ describes the form and position of the free surface. The second equation in (2) is the no-penetration condition on the wetted surfaces of the shell, the third equation here is the kinematics boundary condition, which assumes that a fluid particle of the free surface will always stay on this surface, the forth equation in (2) is the dynamic boundary condition, which consists in equality of the liquid pressure on the free surface to atmospheric one.

So we reduce the problem under consideration to the following system of differential equations:

$$
\mathrm{LU}+\mathrm{MU}=p_{d} \mathrm{n} ; \Delta \Phi=0
$$

with boundary conditions from (2), relative to $\Phi$, and fixation conditions of the shell, relative to $\mathbf{U}$.

\section{THE MODE SUPERPOSITION METHOD FOR COUPLED DYNAMIC PROBLEMS}

Consider modes of fluid-filled shell vibrations in the form

$$
\mathrm{U}=\sum_{k=1}^{N} c_{k}(t) \mathrm{u}_{k}
$$


where $c_{k}(t)$ are unknown coefficients and $\mathbf{u}_{k}$ are the eigenmodes of vibrations of the empty shell. In other words, the mode of vibration of the shell filled by a liquid is determined as a linear combination of eigenmodes of its vibrations without liquid. Due equalities (1) we have

$$
\left(\mathrm{Lu}_{k}, \mathrm{u}_{j}\right)=\Omega_{k}^{2} \delta_{k j},
$$

where $\Omega_{k}$ is the $k$-th frequency of the empty shell vibrations.

Consider the potential $\Phi$ as a sum of two potentials $\Phi=\Phi_{1}+\Phi_{2}$, as it was done by Degtyarev et al. [9].

The series for potential $\Phi_{1}$ can be written as

$$
\Phi_{1}=\sum_{k=1}^{N} \dot{c}_{k}(t) \varphi_{1 k}
$$

Here time-dependant coefficients $c_{k}(t)$ are defined in eqn (4). To determine functions $\varphi_{1 k}$ the following boundary value problems is formulated:

$$
\Delta \varphi_{1 k}=0,\left.\frac{\partial \varphi_{1 k}}{\partial \mathrm{n}}\right|_{\sigma}=w_{k},\left.\varphi_{1 k}\right|_{s_{0}}=0, w_{k}=\left(\mathrm{u}_{k}, \mathrm{n}\right), k=\overline{1, N}
$$

The solution of boundary value problems (5) was done by Ventsel et al. [7].

Thus the dynamic analysis of elastic shells of revolution with a liquid, neglecting the gravity force, is formulated in terms of the functions $\mathbf{U}$ and $\Phi_{1}$. The above functions satisfy the system of differential eqn (3), the no-penetration condition and the lack of the pressure on a free surface, as well as the conditions of the shell fixation. The solutions of the boundary value problems (5) can be represented in the symbolic form as $\varphi_{1 k}=i \Omega \mathrm{H}\left(\mathrm{u}_{k}\right)$, where $\mathrm{H}\left(\mathrm{u}_{k}\right)$ is the inverse operator of the hydrodynamic problem [7].

Suppose that $c_{k}(t)=C_{k} \exp (i \omega t)$, where $\omega$ is an own frequency of the shell with a fluid. Based on the eqns (1), (4) and (5) we obtain

$$
\left(\Omega_{k}^{2} \delta_{k j}+\delta_{k j}\right) C_{j}=\omega^{2} \rho_{l} \sum_{k=1}^{N} C_{k}\left(\mathrm{H}\left(\mathrm{u}_{k}\right), \mathrm{u}_{j}\right) .
$$

The above equation represents a generalized eigenvalue problem. Solving this problem yields the natural frequencies $\omega$ of the vibrations of the elastic shell conveying fluid, but without the gravity effects.

When the potential $\Phi_{2}$ is known, the low frequency sloshing modes will be obtained. To determine the potential $\Phi_{2}$ we have the problem of fluid vibrations in the rigid shell with

gravity effects.
$\quad$ Use the expansion $\Phi_{2}=\sum_{k=1}^{M} d_{k}(t) \varphi_{2 k}$, where $d_{k^{*}}(t)$ - unknown coefficients, functions $\varphi_{2 k}$ are natural modes of the liquid sloshing in the rigid tank. To obtain these modes the following boundary value problems are considered:

$$
\Delta \varphi_{2 k}=0 ;\left.\frac{\partial \varphi_{2 k}}{\partial \mathrm{n}}\right|_{\sigma}=0 \frac{\partial \varphi_{2 k}}{\partial \mathrm{n}}+\left.g \zeta\right|_{s_{0}}=0 ;\left.\frac{\partial \varphi_{2 k}}{\partial \mathrm{n}}\right|_{s_{0}}=\frac{\partial \zeta}{\partial t}, k=\overline{1, N}
$$

Differentiate the third equation in relationship (7) with respect to $t$ and substitute there the expression for $\zeta_{t}^{\prime}$ from the forth one of (7). Suppose $\varphi_{2 k}(t, x, y, z)=e^{i \chi k t} \varphi_{2 k}(x, y, z)$ and obtain the next conditions on the free surface for each mode $\varphi_{2 k}$ with the sloshing frequency $\chi_{k}$ :

$$
\frac{\partial \varphi_{2 k}}{\partial \mathrm{n}}=\frac{\chi_{k}^{2}}{g} \varphi_{2 k}, \quad k=1, M
$$


It leads to the following eigenvalue problems

$$
\Delta \varphi_{2 k}=0 ;\left.\frac{\partial \varphi_{2 k}}{\partial \mathrm{n}}\right|_{\sigma}=0 \quad \frac{\partial \varphi_{2 k}}{\partial \mathrm{n}}=\frac{\chi_{k}^{2}}{g} \varphi_{2 k}, k=1, M .
$$

Solving these problems yields the sloshing frequencies $\chi_{k}$. and modes $\varphi_{2 k}$.

Finally, for the sum of potentials $\Phi=\Phi_{1}+\Phi_{2}$, the following expression can be written

$$
\Phi=\sum_{k=1}^{N} \dot{c}_{k}(t) \varphi_{1 k}+\sum_{k=1}^{M} \dot{d}_{k}(t) \varphi_{2 k}
$$

The unknown function $\zeta$ takes the form

$$
\zeta=\sum_{k=1}^{N} c_{k}(t) \frac{\partial \varphi_{1 k}}{\partial n}+\sum_{k=1}^{M} d_{k}(t) \frac{\partial \varphi_{2 k}}{\partial n} .
$$

To define coupled modes of harmonic vibrations, suppose that $c_{k}(t)=C_{k} \exp (i \omega t)$; $d_{l}(t)=D_{k} \exp (i \omega t)$. Substituting these expressions into eqns (10) and (11) and then into equations

$$
\mathrm{LU}+\mathrm{M} \ddot{\mathrm{U}}=p_{d} \mathrm{n}, \frac{\partial \Phi}{\partial t}+\left.g \zeta\right|_{s_{0}}=0
$$

results in the generalized eigenvalue problem where both elasticity and gravity effects are taken into account [9].

\section{SYSTEMS OF THE BOUNDARY INTEGRAL EQUATIONS AND MULTI-DOMAIN APPROACH}

To define functions $\varphi_{1 k}$ and $\varphi_{2 k}$ we use the boundary element method in its direct formulation Brebbia et al. [10]. Dropping indexes $1 k$ and $2 k$ the main relation can be written in the form

$$
2 \pi \varphi\left(p_{0}\right)=\iint_{s} q \frac{1}{\left|p-p_{0}\right|} d s-\iint_{s} \varphi \frac{\partial}{\partial \mathrm{n}} \frac{1}{\left|p-p_{0}\right|} d s,
$$

where $S=\sigma \cup S_{0}$. The function $\varphi$, defined on the surface $\sigma$, presents the pressure on the wetted shell surface and the function $q$, defined on the surface $S_{0}$, is the flux, $q=\partial \varphi / \partial \mathrm{n}$.

To apply the multi-domain approach we divide the fluid domain into two sub-domains $\Omega_{1}$ and $\Omega_{2}$, shown in Figure 2. Here we introduce the artificial interface surface $S_{\text {int }}$. Let $\sigma_{1}=S_{1} \cup S_{\text {bot }} \cup S_{\text {baf }}$ and $\sigma_{2}=S_{2} \cup S_{\text {baf }}$ are the surfaces of the shell contacting with a liquid in sub-domains $\Omega_{1}$ and $\Omega_{2}$. Then boundaries of sub-domains $\Omega_{1}$ and $\Omega_{2}$ are $\sum_{1}=\sigma_{1} \cup S_{\text {int }}$ and $\sum_{2}=\sigma_{2} \cup S_{0}$.

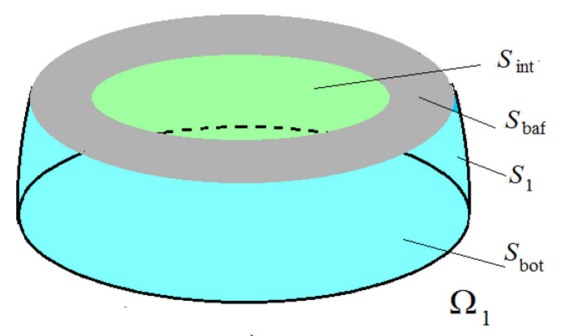

a)

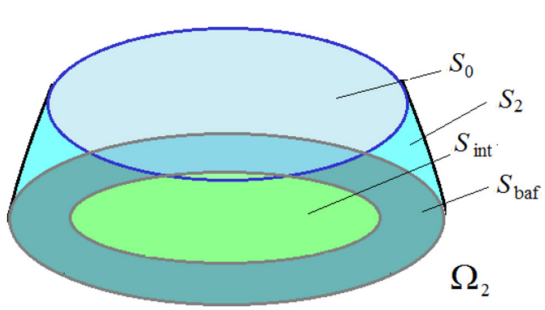

b)

Figure 2: Fluid sub-domains. 
Denote by $\varphi_{1}, \varphi_{2}, \varphi_{0}$ the potential values in nodes of $\sigma_{1}, \sigma_{2}$ and $S_{0}$, respectively. The fluxes on $\sigma_{1}, \sigma_{2}$ are known from the no-penetration boundary condition as $w_{1}, w_{2}$ and on the free surface the unknown flux is denoted as $q_{0}$. The potential and flux values on the interface surface $S_{\text {int }}$ will be unknown functions $\varphi_{j i}$ and $q_{j}, S_{\text {int }} \subset \sum_{j}, j=1,2$, and we have $\varphi_{2 i}=\varphi_{1 i}, q_{1}=-q_{2}$.

Consider the boundary value problem for determining the potential $\Phi_{1}$.

There are two types of kernels in the integral operators introduced above

$$
A(S, \sigma) \psi=\iint_{s} \psi \frac{\partial}{\partial \mathrm{n}} \frac{1}{\left|p-p_{0}\right|} d s ; \quad B(S, \sigma) \psi=\iint_{s} \psi \frac{1}{\left|p-p_{0}\right|} d s ; \quad p_{0} \in \sigma
$$

Introducing $\tilde{S}_{1}=\sigma_{1}, \tilde{S}_{2}=S_{\text {int }}, \tilde{S}_{3}=\sigma_{2}, \tilde{S}_{4}=S_{0}$ allows us to obtain $A_{i j}=A\left(\tilde{S}_{i}, \tilde{S}_{j}\right)$; $B_{i j}=B\left(\tilde{S}_{i}, \tilde{S}_{j}\right) ; i, j=\overline{1,4}$.

By using the multi-domain approach to determine the potential $\Phi_{1}$ the next system of integral equations in the operator form was obtained:

$$
\begin{gathered}
A_{11} \varphi_{1}+A_{12} \varphi_{1 i}=B_{11} w_{1}+B_{12} q_{1} ; \quad p_{0} \in \sigma_{1} ; \\
A_{21} \varphi_{1}+A_{22} \varphi_{1 i}=B_{21} w_{1}+B_{22} q_{1} ; \quad p_{0} \in S_{\mathrm{int}} ; \\
A_{32} \varphi_{1 i}+A_{33} \varphi_{2}=B_{33} w_{2}-B_{32} q_{1}+B_{34} q_{0} ; \quad p_{0} \in \sigma_{2} ; \\
A_{22} \varphi_{1 i}+A_{23} \varphi_{2}=B_{23} w_{2}-B_{22} q_{1}+B_{24} q_{0} ; \quad p_{0} \in S_{\mathrm{int}} ; \\
A_{42} \varphi_{1 i}+A_{43} \varphi_{2}=B_{43} w_{2}-B_{42} q_{1}+B_{44} q_{0} ; \quad p_{0} \in S_{0} .
\end{gathered}
$$

From the first two eqns in (13) the expressions for $\varphi_{1}$ and $\varphi_{1 i}$ were received as functions of fluxes $w_{1}, q_{1}$.

$$
\begin{gathered}
\varphi_{1}=F_{1} w_{1}+F_{2} q_{1} ; \quad \varphi_{1 i}=F_{1 i} w_{1}+F_{2 i} q_{1} \\
F_{1}=A_{\varphi}^{-1} B_{w 1} ; \quad F_{2}=A_{\varphi}^{-1} B_{q 1} ; \quad A_{\varphi}=A_{11}-\left(A_{12} A_{21}\right) / 2 \pi ; \quad B_{w 1}=B_{11}-\left(A_{12} B_{21}\right) / 2 \pi ; \\
B_{q 1}=B_{12}-\left(A_{12} B_{22}\right) / 2 \pi ; \quad F_{1 i}=\left(B_{21}-A_{21} F_{1}\right) / 2 \pi ; \quad F_{2 i}=\left(B_{22}-A_{21} F_{2}\right) / 2 \pi .
\end{gathered}
$$

From third and forth eqns in (13) the analogical expressions were obtained for $\varphi_{2}$ and $\varphi_{1 i}$ as functions of fluxes $w_{2}, q_{1}, q_{0}$. Then $q_{1}$ can be written as

$$
q_{1}=D_{1} w_{1}+D_{2} w_{2}+D_{3} q_{0}
$$

where matrices $D_{1}, D_{2}, D_{3}$ are combinations of $A_{i j}, B_{i j}, i, j=1,2,3$.

Using eqn (15) one can obtain unknowns $\varphi_{2}$ and $\varphi_{1 i}$ as functions of $w_{2}, w_{2}, q_{0}$.

From fifth equation of (13) one can receive $q_{0}$ in the form

$$
q_{0}=H_{1} w_{1}+H_{2} w_{2}
$$

where matrices $H_{1}, H_{2}$ are obtained by using $A_{i j}, B_{i j}, i, j=\overline{1,4}$.

With the above expressions (15), (16) for $q_{0}$ and $q_{1}$, we have

$$
\varphi=\mathrm{Qw}, \quad \varphi=\left.\left\{\varphi_{i}\right\}\right|_{i=1} ^{2} ; \mathrm{W}=\left.\left\{w_{i}\right\}\right|_{i=1} ^{2} ; \quad \mathrm{Q}=\left.\left\{Q_{i j}\right\}\right|_{i, j=1} ^{2},
$$

where $Q_{i j}$ are obtained by using matrices $D_{1}, D_{2}, D_{3}, H_{1}, H_{2}$. 
So for each $\varphi_{1 k}$ the pressure on the surface $\sigma$ will be defined by formulae

$$
p_{k}=-\rho_{l} \ddot{c}_{k}(t) \varphi_{1 k}^{i}(p) ; p \in S_{1} \cup S_{\text {bot }} \cup S_{2} ; p_{k}=-\rho_{l} \ddot{c}_{k}(t)\left[\varphi_{1 k}^{1}(p)-\varphi_{1 k}^{2}(p)\right] ; P \in S_{\text {baf }} .
$$

The boundary value problem for determining the potential $\Phi_{2}$ with multi-domain BEM (MBEM) was solved by Gnitko et al. [11]. The whole fluid domain was divided into several sub-domains for better computational performance than at using the single-domain BEM (SBEM).

\section{SOME NUMERICAL RESULTS}

In this study two kinds of eigenmodes were found by solving the boundary value problems (9) and (6): low frequency sloshing modes and hydroelastic vibration modes. The first ones correspond to the gravity waves on the surface of the liquid, whereas the second ones are the vibration modes of the elastic shell structure modified by the interaction with the liquid.

\subsection{Low frequency sloshing modes}

\subsubsection{Cylindrical shell with baffle}

The partially fluid- filled rigid cylindrical shell with baffles was considered. The parameters of the shell are following: the radius is $R=1 \mathrm{~m}$, the thickness is $h=0.01 \mathrm{~m}$, the length is $L=2 \mathrm{~m}$. The filling level is $H=0.8 \mathrm{~m}$, the baffle position is $H_{1}$, the baffle radius is $R_{\mathrm{b}}=R-R_{\text {int. }}$ (Fig. 1).

Figure 3 demonstrates monotonic dependencies of the first 4 eigenvalues for $\alpha=1$, denoted by $F_{1}, F_{2}, F_{3}, F_{4}$, via the radius of the interface surface $R_{\text {int }}$ at different baffle position $H_{1}, \mathrm{~m}$.
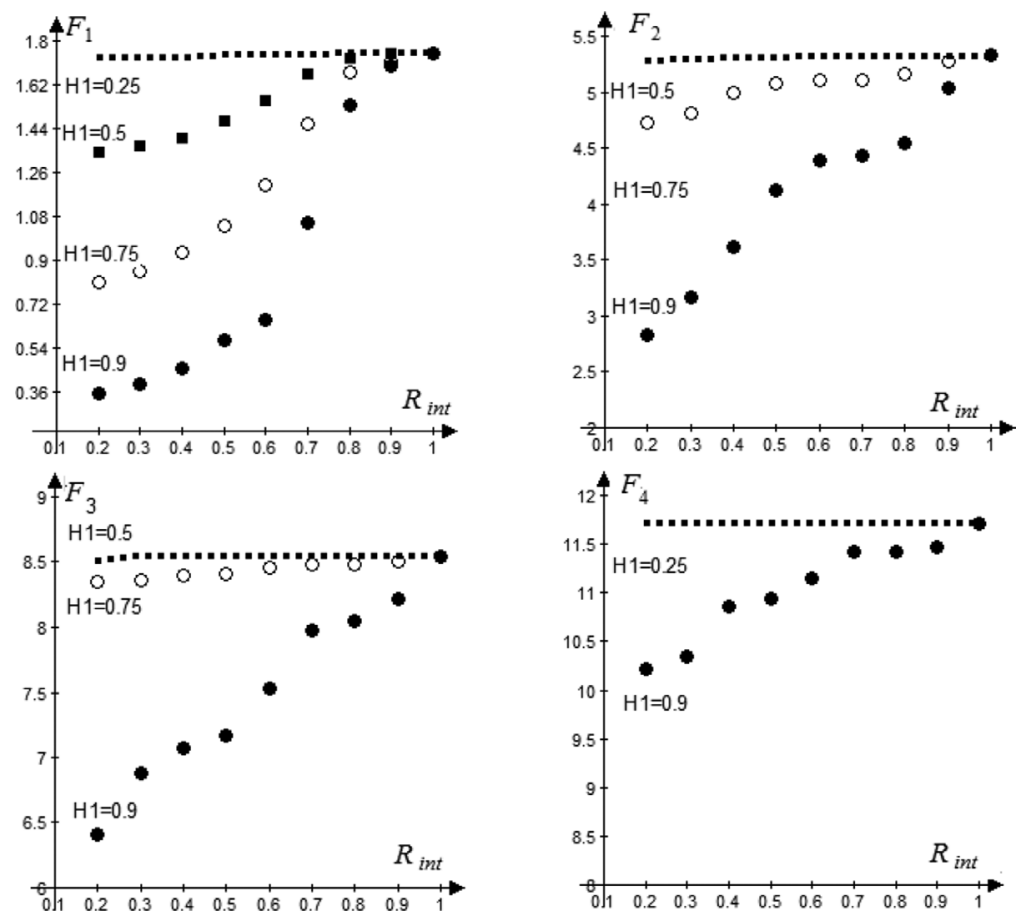

Figure 3: Eigenvalues at $\alpha=1$ versus $R_{\text {int }}$ for $H=1 \mathrm{~m}$ and different $H_{1}$. 


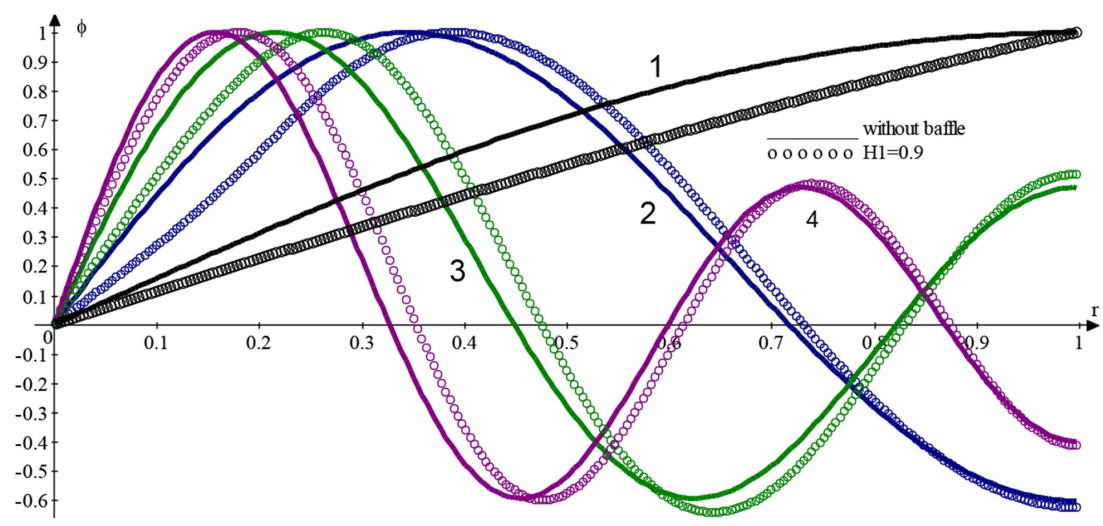

Figure 4: Modes of vibrations of unbaffled and baffled cylindrical tanks, $\alpha=1$.

From these results one can concluded that graphs of $F_{i}$ as functions of $R_{\text {int }}$ are essentially differ for different $i$ and $H_{1}$. The effect of baffles is considerable only for the lower frequencies. Also small baffles (when $R_{\text {int }}$ is relatively large) do not affect the lower frequencies. This conclusion corresponds to results of Gavrilyuk et al. [5]. The liquid above the baffle behaves like a sloshing one while the liquid below the baffle behaves like a rigid one. On the right border of the graphs the values of frequencies coincide with ones obtained for the unbaffled tank.

The first three modes of liquid vibrations are shown on Figure 4.

It should be noted that modes of the shells with and without baffles are similar.

\subsubsection{Conical shell with baffle}

Conical shells in interaction with a fluid have received less attention in scientific literature in spite of the wide usage of thin walled conical shell structures in a number of different branches of engineering.

The numerical procedure for a conical shell in the proposed method is the same as for a cylindrical one. The only distinction consists in formulae for the unit normal and coordinates of considered shell surfaces.

The first estimation was done for unbaffled coextensive cylindrical and conical shells with equal radiuses of free surfaces.

Consider the cylindrical shell with $R_{1}=R_{2}=0.4 \mathrm{~m}$ and $H=3.8464 \mathrm{~m}$. That corresponds to the coextensive $\Lambda$-shape conical shell with $R_{2}=0.4 \mathrm{~m}$ and $R_{1}=1.0 \mathrm{~m}, H=1.0392 \mathrm{~m}$ and $\theta=\pi / 6$. Then consider the cylindrical shell with $R_{1}=R_{2}=1 \mathrm{~m}$ and $H=0.6154 \mathrm{~m}$. That corresponds to the coextensive $V$-shape conical shell with $R_{2}=1 . \mathrm{m}$ and $R_{1}=0.4 \mathrm{~m}, H=1.0392 \mathrm{~m}$ and $\theta=\pi / 6$. Note that for $V$-shape tank the value $R_{1}$ is for the free surface radius, whereas for $\Lambda$-shape tank the value $R_{1}$ is for the radius of bottom.

Table 1 provides the numerical values of the natural liquid sloshing frequencies at $\alpha=1$ for the coextensive cylindrical and conical shells.

The first frequency of cylindrical shell differs essentially for both $\Lambda$ and V-shape tanks. With increasing the frequency number $n$, the difference between results became smaller.

The comparison of our numerical results with data obtained by Gavrilyuk et al. [12] was done. We consider both $V$-shape and $\Lambda$-shape conical tanks with $R_{1}=1 . \mathrm{m}$ and $\theta=\pi / 6$. If $R_{1}$, $R_{2}$ and $\theta$ are known quantities, than the corresponding value of $H$ can be easy found as $H=\left(R_{1}-R_{2}\right) \cot \theta$. In Table 2 the results of numerical simulation are presented for $\alpha=0,1$, 
Table 1: Comparison of frequencies, $\mathrm{Hz}$.

\begin{tabular}{llllll}
\hline Modes & $n=1$ & $n=2$ & $n=3$ & $n=4$ & $n=5$ \\
\hline$\Lambda$-shape conical shell & & & & & \\
Cylinder & 4.6079 & 13.3504 & 21.3866 & 29.3409 & 37.3589 \\
Cone & 5.6206 & 13.9162 & 21.8827 & 29.7942 & 37.6864 \\
V - shape conical shell & & & & \\
Cylinder & 1.4952 & 5.3163 & 8.5358 & 11.7059 & 14.8635 \\
Cone & 1.3052 & 4.9255 & 8.1411 & 11.3169 & 14.6724 \\
\hline
\end{tabular}

Table 2: Natural frequencies of $\mathrm{V}$ - shape and $\Lambda$ - shape conical tanks, $H z$.

\begin{tabular}{lllllllll}
\hline \multicolumn{7}{c}{ V-shape } & \multicolumn{7}{c}{$\Lambda$-shape } \\
\hline$R_{2}$ & 0.2 & 0.6 & 0.8 & 0.9 & 0.2 & 0.6 & 0.8 & 0.9 \\
\hline$\alpha=0, n=1$ & & & & & & & \\
{$[12]$} & 3.386 & 3.382 & 3.139 & 2.187 & 24.153 & 6.669 & 4.545 & 2.678 \\
MBEM & 3.389 & 3.391 & 3.192 & 2.200 & 20.027 & 6.669 & 4.545 & 2.678 \\
$\alpha=1, n=1$ & & & & & & & \\
{$[12]$} & 1.304 & 1.254 & 0.934 & 0.542 & 11.332 & 3.515 & 1.661 & 0.726 \\
MBEM & 1.305 & 1.259 & 0.954 & 0.574 & 11.303 & 3.481 & 1.651 & 0.732 \\
$\alpha=2, n=1$ & & & & & & & \\
{$[14]$} & 2.263 & 2.255 & 2.015 & 1.361 & 17.760 & 8.967 & 5.941 & 3.724 \\
MBEM & 2.265 & 2.269 & 2.048 & 1.394 & 17.939 & 8.965 & 5.941 & 3.726 \\
\hline
\end{tabular}

$2 ; n=1$ and different values of $R_{2}$ in comparison with data [12]. The results are in good agreement except for the frequency of $\Lambda$ - shape tank at $\alpha=0$ and $R_{2}=0.2 \mathrm{~m}$. But it was noted in [12] that in this case the low convergence was achieved by the proposed analytical method.

The numerical simulation of the natural frequencies of liquid sloshing for both $\mathrm{V}$ - shape and $\Lambda$ - shape conical tanks was carried out for different $n$ and $\alpha$. Here $H=H_{1}+H_{2}=1.0 \mathrm{~m}$, $R_{1}=1.0 \mathrm{~m}, R_{2}=0.5 . \mathrm{m}$ for both type of tanks, $H_{1}$ is the baffle positions (Fig. 5 ).

In Table 3 the results of numerical simulation are presented for $\alpha=0,1$ and $n=1, . ., 4$ and different $H_{1}, H_{2}, R_{\text {int }} / R_{\mathrm{b}}$.

Natural frequencies of $\mathrm{V}$ - shape and $\Lambda$ - shape conical tanks without baffles correspond to values $H_{1}=H_{2}=0.5 \mathrm{~m}, R_{\text {int }} / R_{\mathrm{b}}=1$.

Results obtained show different behaviour for $\mathrm{V}$ - shape and $\Lambda$ - shape conical tanks. For $\Lambda$ - shape tanks the baffle positions and their sizes are not affected essentially on the frequencies, while for $\mathrm{V}$ - shape tanks these effects are more considerable.

5.2 Hydroelastic vibration modes for baffled cylindrical shell

The elasticity effects of both baffles and tank walls were considered in studying the effects of fluid-structure interaction. We considered the cylindrical shell with a flat bottom, with $R=1 \mathrm{~m}, h=0.01 \mathrm{~m}, L=2 \mathrm{~m}$, Young's modulus $E=2 \cdot 10^{5} \mathrm{MPa}$, Poisson's ratio $v=0.3$. The 

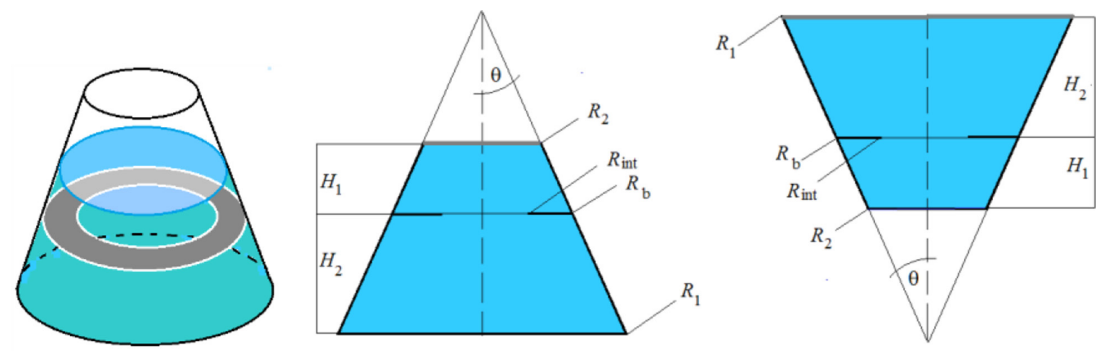

Figure 5: Baffled conical shells of $\Lambda$ and V shapes.

Table 3: Natural frequencies of $\mathrm{V}$ - shape and $\Lambda$ - shape baffled conical tanks, $H z$.

\begin{tabular}{llllllllllll}
\hline & & & \multicolumn{4}{c}{ V-shape tank } \\
\cline { 4 - 12 }$H_{1}$ & $H_{2}$ & $R_{\text {int }} / R_{\mathrm{b}}$ & \multicolumn{1}{c}{$\Lambda$-shape tank } \\
\hline 0.5 & 0.5 & 1 & 3.466 & 6.681 & 9.845 & 12.99 & 7.985 & 14.37 & 20.70 & 27.01 \\
0.5 & 0.5 & 0.5 & 3.408 & 6.668 & 9.843 & 12.99 & 7.968 & 14.37 & 20.69 & 27.01 \\
0.8 & 0.2 & 0.2 & 2.443 & 6.059 & 9.565 & 12.88 & 7.113 & 14.20 & 20.65 & 26.99 \\
& & & $\alpha=1$ & & & & & & & \\
0.5 & 0.5 & 1 & 1.416 & 4.997 & 8.206 & 11.37 & 4.424 & 11.09 & 17.46 & 23.79 \\
0.5 & 0.5 & 0.5 & 1.228 & 4.974 & 8.197 & 11.37 & 4.192 & 11.06 & 17.46 & 23.79 \\
0.8 & 0.2 & 0.2 & 0.630 & 4.191 & 7.849 & 11.23 & 2.529 & 10.66 & 17.36 & 23.75 \\
\hline
\end{tabular}

Table 4: Frequencies of elastic cylindrical shells with baffles, $H z$.

\begin{tabular}{lllllllll}
\hline$n$ & \multicolumn{3}{l}{ Empty elastic shell } & \multicolumn{5}{c}{ Fluid-filled elastic shell } \\
\cline { 2 - 9 }$R_{b}$ & 0 & 0.2 & 0.5 & 0.8 & 0 & 0.2 & 0.5 & 0.8 \\
\hline 1 & 23.233 & 23.233 & 23.234 & 23.234 & 7.9259 & 7.5901 & 5.5213 & 1.7874 \\
2 & 91.1011 & 91.1014 & 40.4818 & 24.4105 & 43.3566 & 42.350 & 15.172 & 9.7932 \\
3 & 205.252 & 192.172 & 91.1015 & 91.1016 & 117.034 & 116.02 & 46.769 & 45.914 \\
4 & 365.795 & 205.253 & 205.253 & 100.789 & 230.316 & 228.95 & 119.14 & 52.908 \\
5 & 392.787 & 365.795 & 214.258 & 205.253 & 392.787 & 229.18 & 168.05 & 119.77 \\
\hline
\end{tabular}

material's density is $\rho_{s}=7,800 \mathrm{~kg} / \mathrm{m}^{3}$, the fluid density is $\rho_{l}=1,000 \mathrm{~kg} / \mathrm{m}^{3}$, the filling level is $H=0.8 \mathrm{~m}$. In Table 4 the frequencies of axisymmetric vivrations are presented. The baffle position is $H_{1}=0.45 \mathrm{~m}$, the baffle radius $R_{b}$ was variable. The value $R_{b}=0$ corresponds to the unbaffled tank.

From these results one can concluded that both elasticity effects and the size of baffles are affect essentially on the values of natural frequencies.

\section{CONCLUSIONS}

The multi-domain boundary element method has been implemented to solve the problems of hydro-elastic vibrations without neglecting gravity effects. The proposed approach allows us 
to carry out the numerical simulation of vibrations for tanks with baffles of different sizes and positions in the tank. This gives the possibility of governing the baffle radius and its position within the tank by using numerical simulation. The rigid and elastic baffled tanks were considered. Dependencies of frequencies via the filling level were obtained numerically for vibrations of the fluid-filled cylindrical and conical tanks with and without baffles. The geometry of tank can be easy changed without complicated analytical calculations. The elasticity effects were considered.

\section{ACKNOWLEDGMENTS}

The authors gratefully acknowledge Professor Carlos Brebbia, Wessex Institute of Technology, for his constant support and interest to our research.

\section{REFERENCES}

[1] Popov, G., Sankar, S. \& Sankar, T.S., Dynamics of liquid sloshing in baffled and compartmented road containers. Journal of Fluids and Structures, 7, pp. 803-821, 1993. http://dx.doi.org/10.1006/jfls.1993.1047

[2] Guorong, Y. \& Rakheja, S., Straight-line braking dynamic analysis of a partly-filled baffled and unbaffled tank truck. Proceedings of the Institution of Mechanical Engineers, Part D: Journal of Automobile Engineering, 223, pp. 11-26, 2009. http://dx.doi.org/10.1243/09544070JAUTO973

[3] Lloyd, N., Vaiciurgis, E. \& Langrish, T.A.G., The effect of baffle design on longitudinal liquid movement in road tankers: an experimental investigation. Process Safety and Environment Protection, 80(4), pp. 181-185, 2002. http://dx.doi.org/10.1205/095758202320439137

[4] Bermudez, A. \& Rodrigues, R., Finite element analysis of sloshing and hydroelastic vibrations under gravity. Mathematical Modelling and Numerical Analysis, 33(2), pp. 305-327, 1999.

http://dx.doi.org/10.1051/m2an:1999117

[5] Gavrilyuk, I., Lukovsky, I., Trotsenko, Yu. \& Timokha, A., Sloshing in a vertical circular cylindrical tank with an annular baffle. Part 1. Linear fundamental solutions. Journal of Engineering Mathematics, 54, pp. 71-88, 2006. http://dx.doi.org/10.1007/s10665-005-9001-6

[6] Levitin M. \& Vassiliev D., Vibrations of shells contacting fluid: asymptotic analysis. In Acoustic Interactions with Submerged Elastic Structures, eds A. Guran, J. Ripoche \& F. Ziegler, World Scientific, 5, pp. 310-332, 1996. http://dx.doi.org/10.1142/9789812830593_0010

[7] Ventsel, E., Naumenko, V., Strelnikova, E. \& Yeseleva, E., Free vibrations of shells of revolution filled with a fluid. Engineering Analysis with Boundary Elements, 34, pp. 856-862, 2010.

http://dx.doi.org/10.1016/j.enganabound.2010.05.004

[8] Lamb, H., Hydrodynamics, 6th edn., Cambridge University Press, 1993.

[9] Degtyarev, K., Glushich, P., Gnitko, V. \& Strelnikova, E., Numerical simulation of free liquid-induced vibrations in elastic shells. International Journal of Modern Physics and Applications, 1(4), pp. 159-168, 2015.

[10] Brebbia, C.A., Telles, J.C.F. \& Wrobel, L.C., Boundary Element Techniques, SpringerVerlag: Berlin and New York, 1984. http://dx.doi.org/10.1007/978-3-642-48860-3 
[11] Gnitko, V., Naumenko, V., Rozova, L. \& Strelnikova, E., Multi-domain boundary element method for liquid sloshing analysis of tanks with baffles. Journal of Basic and Applied Research International, 17(1), pp. 75-87, 2016.

[12] Gavrilyuk, I., Hermann, M., Lukovsky, I., Solodun, O. \& Timokha, A., Natural sloshing frequencies in truncated conical tanks. Engineering Computations, 25(6), pp. 518-540, 2008.

http://dx.doi.org/10.1108/02644400810891535 